\title{
Identification of Lacrimal Gland Postganglionic Innervation and Its Regulation of Tear Secretion
}

Kai Jin, ${ }^{*}$ Toshihiro Imada, ${ }^{*}$ Ryuji Hisamura, ${ }^{*}$ Masataka Ito, ${ }^{\dagger}$ Haruki Toriumi, ${ }^{\ddagger}$ Kenji F. Tanaka, ${ }^{\S}$ Shigeru Nakamura, ${ }^{*}$ and Kazuo Tsubota*

From the Departments of Ophthalmology, ${ }^{*}$ Neurology, ${ }^{\ddagger}$ and Neuropsychiatry, ${ }^{\S}$ Keio University School of Medicine, Tokyo; and the Department of Developmental Anatomy and Regenerative Biology, ${ }^{\dagger}$ National Defense Medical College, Saitama, Japan

Accepted for publication

January 7, 2020.

Address correspondence to Shigeru Nakamura, D.V.M., Ph.D., or Kazuo Tsubota, M.D. Ph.D., Department of Ophthalmology, Keio University School of Medicine, 35 Shinanomachi, Shinjuku-ku, Tokyo 160-8582, Japan. E-mail: s-nakamura.a5@keio.jp or tsubota@z3.keio.jp.

\begin{abstract}
Tear fluid secreted from the exocrine lacrimal gland (LG) has an essential role in maintaining a homeostatic environment for a healthy ocular surface. Tear secretion is regulated by the sympathetic and parasympathetic components of the autonomic nervous system, although the contribution of each component is not fully understood. To investigate LG innervation, we identified sympathetic and parasympathetic postganglionic nerves, specifically innervating the mouse $L G$, by injecting a retrograde neuronal tracer into the LG. Interruption of neural stimuli to the LG by the denervation of these postganglionic nerves immediately and chronically decreased tear secretion, leading to LG atrophy along with destruction of the lobular structure. This investigation also found that parasympathetic, but not sympathetic, innervation was involved in these alterations. (Am J Pathol 2020, 190: 1068-1079; https://doi.org/10.1016/j.ajpath.2020.01.007)
\end{abstract}

Tears comprise a serous body fluid that constantly covers and moistens the ocular surface and have two secretory modes and roles. Basal secretion, a continuous low-level flow, covers the avascular cornea and helps in maintaining a healthy, transparent ocular surface by providing a proper homeostatic balance. ${ }^{1}$ Reflex tearing, a marked transient secretion caused by mechanical stimulation of the ocular surface, emoves foreign bodies or irritant chemicals from the ocular surface. ${ }^{1,2}$ Recent findings revealed that tears also act as a pheromone, necessary for instinctive human social behaviors. ${ }^{1,3}$ One of the most common complications of tear functional status is dry eye syndrome. It is a multifactorial disease that involves tear function and significantly affects quality of life by being accompanied with discomfort, visual disturbance, and tear film instability, with potential damage to the ocular surface. ${ }^{4}$ It was previously reported, in a human epidemiological study and in a rat dry eye model, that insufficient tear secretion contributes to the etiology of technological device-associated dry eye, ${ }^{5,6}$ of which reduced blinking while gazing has been considered to be a major causative factor. Blinking is a spontaneous action whose purpose is not only to protect the eye from foreign body insults and excess light ${ }^{7,8}$ but also to maintain basal tear secretion by transmitting sensory signals of lid wiper friction through the sensory and autonomous nervous system. ${ }^{8,9}$ The cause of this type of dry eye has not yet been clarified, although the involvement of lacrimal gland (LG) innervation has been proposed. ${ }^{6,10}$

Tear secretion is precisely regulated by the coordination of the central nervous system, and LG activity is controlled by sensory, sympathetic, and parasympathetic nerves. ${ }^{2,11,12}$ Sensory-evoked afferent signals from the ocular surface, such as mechanical stimulation by spontaneous blinking and environmental stimulation, are transmitted to the central nervous system via trigeminal nerves. These sensory signals are transmitted to the efferent sympathetic and parasympathetic pathways, which reach the LG and regulate tear secretion. The sympathetic pathway originates from the intermediolateral cell column in the thoracic spinal cord, and the parasympathetic pathway originates from the superior salivatory nucleus in the pons. Sympathetic and

Supported by Japan Society for the Promotion of Science (JSPS) Grantsin-Aid for Scientific Research (KAKENHI) (grant 17K16983 to T.I.).

K.J. and T.I. contributed equally to this work.

Disclosures: None declared. 
parasympathetic nerves pass through the superior cervical ganglion and the pterygopalatine ganglion, respectively, and get distributed around the facial area where the LG is located. ${ }^{13}$ A retrograde tracing study on the distribution of preganglionic neurons from the LG of cynomolgus monkeys found that the retrograde tracer is transported to superior cervical ganglion and pterygopalatine ganglion neurons. ${ }^{14}$

The role of sympathetic innervation in the secretory function of the LG remains controversial. Tangkrisanavinont ${ }^{15}$ reported that electrostimulation of the superior cervical ganglion increases tear secretion, but no alteration of tear secretion following superior cervical ganglion electrostimulation was noted by Botelho et al. ${ }^{16}$ Studies on the functional interaction between the pterygopalatine ganglion and the LG found that tear secretion is abolished and elevated, respectively, by surgical denervation ${ }^{17}$ and electrostimulation of the pterygopalatine ganglion. ${ }^{16}$ However, it is unclear whether these changes in tear secretion induced by pterygopalatine ganglion denervation or stimulation are directly mediated by the LG because several studies suggested that the pterygopalatine ganglion sends projections to diverse orbital targets other than the LG, such as the nasal and palatal mucosa. ${ }^{18-20}$ Salvatore et $\mathrm{al}^{21}$ determined the site of postganglionic nerve entry to the LG by anterograde dissection from the rabbit trigeminal ganglion and found that tear composition was altered by distal postganglionic denervation (PGD). Although the anatomical and physiologic roles of LG innervation have been extensively investigated by various researchers during the past few decades, no previous study provides a comprehensive understanding of the role of LG innervation in the functional and morphologic regulation of the LG.

This study identified the distribution and routes of the postganglionic nerves specifically innervating the LG by a combination of retrograde neural labeling and light sheet microscopy, allowing for whole organ imaging with spatiotemporal resolution. To clarify the exact role of the autonomic nerve innervation of the LG, this study examined the contribution of the postganglionic nerves identified to be directly entering the LG to the regulation of LG secretory function and morphology by using PGD combined with pharmacologic approaches.

\section{Material and Methods}

\section{Animals}

Female C57BL/6 mice (CLEA Japan, Tokyo, Japan), aged 8 weeks, were used in this study. All animals were quarantined and acclimatized for 1 week before the experiments under the following general conditions: room temperature of $23^{\circ} \mathrm{C} \pm 2^{\circ} \mathrm{C}$, relative humidity of $60 \% \pm 10 \%$, alternating 12 hour light-dark cycle (8 AM to $8 \mathrm{PM}$ ), and free access to water and food. All animals were used according to the Association of Research and Vision in Ophthalmology statement for the Use of Animals in Ophthalmic and Vision Research. (https:// www.arvo.org/about/policies/statement-for-the-use-of-animalsin-ophthalmic-and-vision-research, last accessed April 13, 2020) The protocol for this study was approved by the Ethics Committee on Animal Research of the Keio University School of Medicine (approval number 12111-1). To visualize the peripheral nerve projection into the LG, the investigators used transgenic mice that expressed yellow fluorescent protein (YFP) in the Schwann cells [proteolipid protein (PLP) - tetracycline-controlled transcriptional activator (tTA)::tetO-ChR2-EYFP double transgenic ${ }^{22}$ (PLP-YFP)].

\section{Retrograde Labeling with Cholera Toxin Subunit B}

After mice were deeply anesthetized with a combination anesthetic that contained medetomidine $(0.75 \mathrm{mg} / \mathrm{kg})$, butorphanol $(5 \mathrm{mg} / \mathrm{kg})$, and midazolam $(4 \mathrm{mg} / \mathrm{kg})$, the facial skin above the exorbital LG was carefully incised (1-cm incision) with small straight scissors, and the LG was denuded. A retrograde neuronal tracer, Alexa 555-conjugated cholera toxin subunit B (CTB; $0.3 \mu \mathrm{L}$ of $0.1 \%$; Life Technologies, Carlbad, CA) was injected into the LG using the Hamilton microsyringe (Hamilton, Reno, NV), and then the incised site was sutured. Fluorescent observation of the LG was performed by light sheet microscopy (Carl Zeiss, Munich, Germany) at 3 days after CTB injection to examine the CTB-labeled nerve fibers. Three-dimensional reconstruction of CTB-labeled nerve fibers was performed using Imaris software version 7.7.1 (Bitplane, Zurich, Switzerland).

\section{PGD Surgery}

Surgical procedures were performed with the mice under deep general anesthesia with i.p. injections of a combination anesthetic that contained medetomidine $(0.75 \mathrm{mg} / \mathrm{kg})$, butorphanol $(5 \mathrm{mg} / \mathrm{kg})$, and midazolam $(4 \mathrm{mg} / \mathrm{kg})$. Denervation surgery was performed at the caudal root site of the nerve bundle along the blood vessels (PGD). After a nylon thread was hung on this nerve bundle (Supplemental Figure S1A), both ends of the thread were passed inside the polyethylene tube. The nerve bundle was cut by pulling these ends.

\section{Superior Cervical Ganglion Ablation}

Mice were placed in the supine position after being deeply anesthetized with sodium pentobarbital $(64.8 \mathrm{mg} / \mathrm{kg}$ intraperitonally). The mice cervical skin above the submandibular gland was carefully incised for $2 \mathrm{~cm}$ with small straight scissors. The superior cervical ganglion, which is adjacent to the carotid artery and located at the medial side of submanidibular gland (Supplemental Figure S2A), was ablated by the batterypowered cautery, and then the incised site was sutured.

\section{Measurement of Tear Secretion}

A modified Schirmer test was used on mouse eyes to measure basal tear secretion. ${ }^{23}$ A phenol red thread (Showa 
Yakuhin Kako, Tokyo, Japan) was placed on the temporal side of the upper eyelid margin for 15 seconds. The length of the moistened area from the edge was measured with a precision of $0.5 \mathrm{~mm}$. Stimulated tear secretion was measured 5 minutes after the i.p. injection of carbachol (cholinergic agent, $0.1 \mathrm{mg} / \mathrm{kg}$; Tokyo Chemical Industry, Tokyo, Japan) or immediately after repeated irritation of the cornea with nylon thread (physical stimulation).

\section{Corneal Fluorescein Staining}

Changes in the corneal surface were determined by corneal fluorescein staining under a blue-free barrier filter. ${ }^{24}$ Mice were anesthetized with a combination anesthetic that contained medetomidine $(0.75 \mathrm{mg} / \mathrm{kg})$, butorphanol $(5 \mathrm{mg} / \mathrm{kg})$, and midazolam $(4 \mathrm{mg} / \mathrm{kg})$, and then $1 \mu \mathrm{L}$ of $0.5 \%$ fluorescein sodium solution was instilled into the conjunctival sac. After washing away the excess fluorescein sodium solution, observation of the cornea was performed using a fluorescent stereomicroscope (M205FA; Leica, Hamburg, Germany).

\section{Blood Flow Analysis}

To investigate whether PGD changes LG blood flow, blood flow was measured using a laser Doppler flow meter (ALF21R, Advance, Japan) with LabChart software version 6 (ADInstruments, Sydney, Australia). ${ }^{25}$ An $0.8 \mathrm{~mm}$ probe, was placed on the upper margin of the medial surface of the LG after each mouse was deeply anesthetized with sodium pentobarbital $(64.8 \mathrm{mg} / \mathrm{kg}$ intraperitoneally). PGD was performed carefully without touching the probe. LG blood flow was measured before and after PGD for 2 minutes each.

\section{Histopathologic Examination}

Mice were euthanized at 3 days after CTB injection into the LG, with or without PGD. Mice were anesthetized with sodium pentobarbital $(120 \mathrm{mg} / \mathrm{kg}$ intraperitoneally) and sacrificed by exsanguination by cutting the carotid artery. The heads or LGs of each animal were removed immediately after death. A multipurpose cryosection preparation kit (Section Lab, Hiroshima, Japan) was used for preparing whole head or LG sections. ${ }^{26}$ The whole head or the LG were frozen in isopentane $\left(-160^{\circ} \mathrm{C}\right)$, cooled with dry ice, and then freeze-embedded with SCEM (Suzuki Composite Electro-chemical Material) compound (Section Lab). Transverse sections $10 \mu \mathrm{m}$ thick were cut with a cryomicrotome (CM3050S, Leica) around the apparatus of external auditory cannula and the posterior eyelid corner for the examination of superior cervical ganglion (Supplemental Figure S3A) and pterygopalatine ganglion, respectively (Supplemental Figure S3B), and collected with cryofilms (Section Lab). For immunohistochemistry, the sections were incubated with primary antibodies to choline transporter-1 (parasympathetic marker, rabbit polyclonal, 1:200 dilution,
Frontier Institute, Hokkaido, Japan), norepinephrine transporter (sympathetic marker, goat polyclonal, 1:200 dilution, Frontier Institute), and calponin (myoepithelial marker, rabbit monoclonal, 1:200 dilution; Abcam, Cambridge, UK) at $4^{\circ} \mathrm{C}$ overnight, followed by labeling with Alexaconjugated species-specific secondary antibody (1:400 dilution; Life Technologies) at room temperature for 30 minutes. Hoechst 33342 (1:100 dilution; Dojindo, Kumamoto, Japan) and/or phalloidin (1:40 dilution; Life Technologies) were used as counterstains.

For hematoxylin and eosin staining, LGs were fixed in $10 \%$ formalin, embedded in paraffin, and sectioned at $4 \mu \mathrm{m}$ of thickness. Sections were subjected to hematoxylin and eosin staining. Acinar cell areas were analyzed using ImageJ version 1.52a (NIH, Bethesda, MD; http://imagej.nih.gov/ij) after three random histologic fields per section were acquired from one LG tissue per mouse. Each group consisted of three mice. Images were captured with a microscope (BZ-9000; Keyence, Osaka, Japan) or a confocal microscope (LSM 710; Carl Zeiss).

\section{Transmission Electron Microscopy}

Each mouse was perfused with Karnovsky's fixative (2.5\% glutaraldehyde and $2 \%$ paraformaldehyde in $0.1 \mathrm{~mol} / \mathrm{L}$ sodium cacodylate; $\mathrm{pH}$ 7.4) while under anesthesia. After the LGs were removed from the carcass, they were immersed in the same fixative at $4^{\circ} \mathrm{C}$. Sections of $1-\mu \mathrm{m}$ thickness were stained with methylene blue, and thin sectioning was performed with a diamond knife. The sections were collected on mesh grids, stained with uranyl acetate and lead citrate, and examined with transmission electron microscopy (TEM; 1230 EXII, JEOL, Akishima, Japan). All images were obtained with a bio scan camera (model 792; Gatan, Tokyo, Japan).

\section{Parasympathetic Agonist and Antagonist Infusion}

Continuous infusion of a sympathetic agonist, norepinephrine (Sigma-Aldrich, St. Louis, MO), or a parasympathetic agonist, carbachol (both muscarinic and nicotinic acetylcholine receptor agonists) and bethanechol (muscarinic acetylcholine receptor agonist; Sigma-Aldrich), was performed 1 day after PGD surgery using an osmotic pump $(0.24 \mu \mathrm{L} /$ hour for 7 days; Alzet, Cupertino, CA), which was implanted under the dorsal skin of the mice while under inhalation anesthesia using isoflurane. These agonists were appropriately prepared in saline solution and their dosage was designed for continuous infusion at $0.2 \mathrm{mg} / \mathrm{kg}$ per day.

Continuous infusion of sympathetic antagonists, phenoxybenzamine ( $\alpha$-receptor antagonist; Tokyo Chemical, Tokyo, Japan) and propranolol ( $\beta$-receptor antagonist; Tokyo Chemical), or a parasympathetic antagonist, scopolamine (muscarinic acetylcholine receptor antagonist; Tokyo Chemical) was performed in the normal state. The osmotic pumps $(0.24 \mu \mathrm{L} /$ hour for 7 days; Alzet) that contained these 
antagonists were implanted under the dorsal skin of the mice while under inhalation anesthesia using isoflurane. These antagonists were appropriately prepared in saline solution, and their dosage was designed for continuous infusion at 7.2 $\mu \mathrm{g} / \mathrm{kg}$ per day.

\section{Western Blot Assays}

Mice were euthanized with an overdose of pentobarbital sodium. The excised whole LG was homogenized with a Polytron homogenizer (PT 1200E; Kinematica, Luzern, Switzerland) for 1 minute in a radioimmunoprecipitation assay buffer $(50 \mathrm{mmol} / \mathrm{L}$ Tris hydrochloride $\mathrm{pH} 7.5,150$ $\mathrm{mmol} / \mathrm{L}$ sodium chloride, $1.0 \%$ Igepal CA-630) that contained a protease inhibitor cocktail (Complete Mini; Roche Diagnostic, Rotkreuz, Switzerland). Samples were centrifuged at $4^{\circ} \mathrm{C}$ and $14,000 \times g$ for 5 minutes, and the protein concentration in each LG homogenate was determined using a DC-protein assay kit (Bio-Rad, Hercules, CA). Next, the same volume of $2 \times$ Laemmli sample buffer was added, together with 5\% $\beta$-mercaptoethanol. After boiling, samples were separated by polyacrylamide gel electrophoresis, transferred to polyvinylidene difluoride membranes using a dry blotting system (V20-SDB; SCIE-PLAS, Cambridge, UK), and incubated with primary antibodies to the mammalian target of rapamycin (mTOR, rabbit polyclonal, 1:1000 dilution; Cell Signaling Technology, Tokyo, Japan), phospho-mTOR (p-mTOR, rabbit polyclonal, 1:1000 dilution; Cell Signaling Technology), ribosomal protein S6 (S6, rabbit polyclonal, 1:1000 dilution; Cell Signaling Technology), phospho-S6 (p-S6, rabbit polyclonal, 1:1000 dilution; Cell Signaling Technology), p62 (rabbit polyclonal, 1:1000 dilution; Medical and Biological Laboratories, Nagoya, Japan), microtubuleassociated protein 1 light chain 3 (LC3, rabbit polyclonal, 1:1000 dilution; Novus Biologicals, Centennial, CO), or mouse polyclonal antibody against $\beta$-actin (mouse monoclonal, 1:2000 dilution; Sigma-Aldrich). Then alkaline phosphatase-conjugated species-specific secondary antibodies were adopted before treatment with the 5-bromo-4chloro-3-indolyl-phosphate/nitro blue tetrazolium substrate solution. The Western blot intensities were subsequently analyzed by ImageJ.

\section{Measurement of Reactive 0xygen Species Production and ATP Content}

Mice were euthanized with an overdose of pentobarbital sodium. The excised whole LG was immersed in cold phosphate-buffered saline ( $25 \mathrm{mg} / \mathrm{mL}$ of tissue weight) and homogenized using a Mil mixer with a zirconia ball (AS ONE Corporation, Osaka, Japan) for 3 minutes. Homogenized LGs were examined to measure the reactive oxygen species (ROS) formation and ATP content. ROS levels were measured using the ROS-sensitive fluorescence indicator $2^{\prime}, 7^{\prime}$-dichlorofluorescein diacetate (Molecular Probes,
Eugene, OR). LG homogenates were incubated with $2^{\prime}, 7^{\prime}$ dichlorofluorescein diacetate for 1 hour at $37^{\circ} \mathrm{C}$. The preparations were washed three times with phosphate-buffered saline by centrifugation at $800 \times g$ for 3 minutes. Washed cells were resuspended in phosphate-buffered saline, followed up, and examined at 480-nm excitation and 530-nm emission. To measure the ATP content, LG homogenates were incubated for 5 minutes at $98^{\circ} \mathrm{C}$. The preparations were centrifuged at $800 \times g$ for 3 minutes, and the supernatant was used for ATP measurement. ATP content was determined using the chemiluminescence method (ATP Bioluminescence Assay Kit CLS 2; Roche Molecular Biochemicals, Mannheim, Germany). ATP calibration was performed using ATP and luciferase. Each measurement was performed with a microplate reader (Synergy 4; Biotek Company, Winooski, VT).

\section{Statistical Analysis}

Statistical analyses were performed using JMP software version 12 (SAS Institute Inc., Cary, NC). Comparisons between the two groups were performed with the $U$-test, and multiple comparisons were performed with the Steel test. Differences among the measurement variables were considered significant if the resultant $P \leq 0.05$. All data in this study were collected from scientifically designed randomized experiments.

\section{Results}

Identification of Postganglionic Nerves Projecting to the $L G$

The mouse LG is a flat glandular tissue located inferior to the ear on the surface of the subcutaneous masseter muscle (Figure 1A). To visualize the distribution of postganglionic nerves projecting to the LG, we conducted bright field and YFP observations of the LG in the PLP-YFP mouse. On the lateral view of the LG, YFP-positive nerve bundles were not observed on the lateral surface of the LG surrounding the lacrimal duct going out from the rostral side of the LG (Figure 1B). On the medial view of the LG, two YFPpositive nerve bundles were observed. One was localized along the lacrimal duct, from dorsal to ventral, rostral to the LG, and the other was localized along the blood vessels from dorsal to ventral, caudal to the LG, with a bifurcation at the dorsal end (Figure 1B). These results indicate that two nerve bundles are distributed along both the rostral lacrimal duct and the caudal blood vessels in the medial surface of the LG.

To clarify which nerve bundles have more projection into the LG, the retrograde neuronal tracer CTB was injected into the LG. No CTB-labeled nerve bundles were observed in the lateral surface or rostrally in the medial surface of the LG (data not shown). CTB-labeled nerve fibers, which ramify from the dorsal to the ventral part, 
A

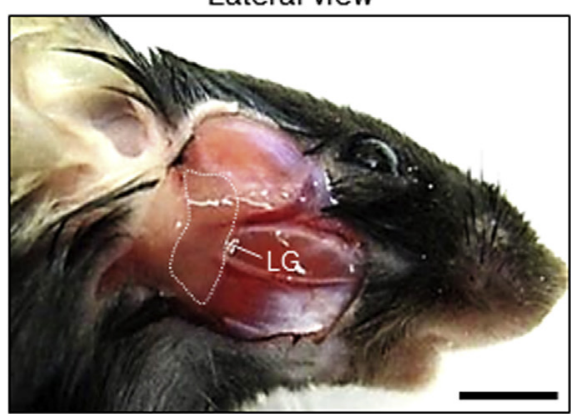

Schematic drawing

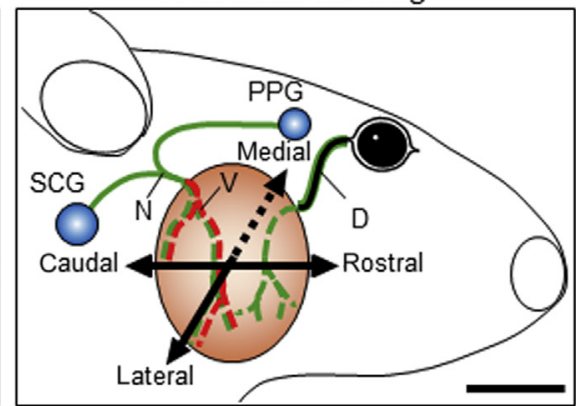

C

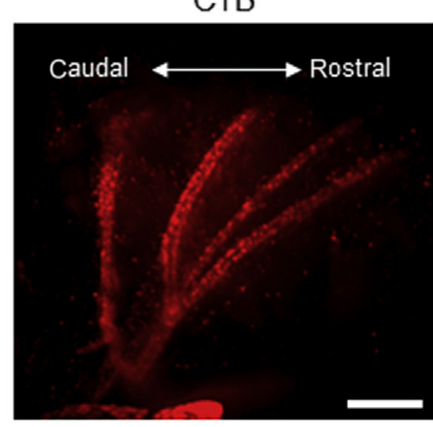

Schematic drawing
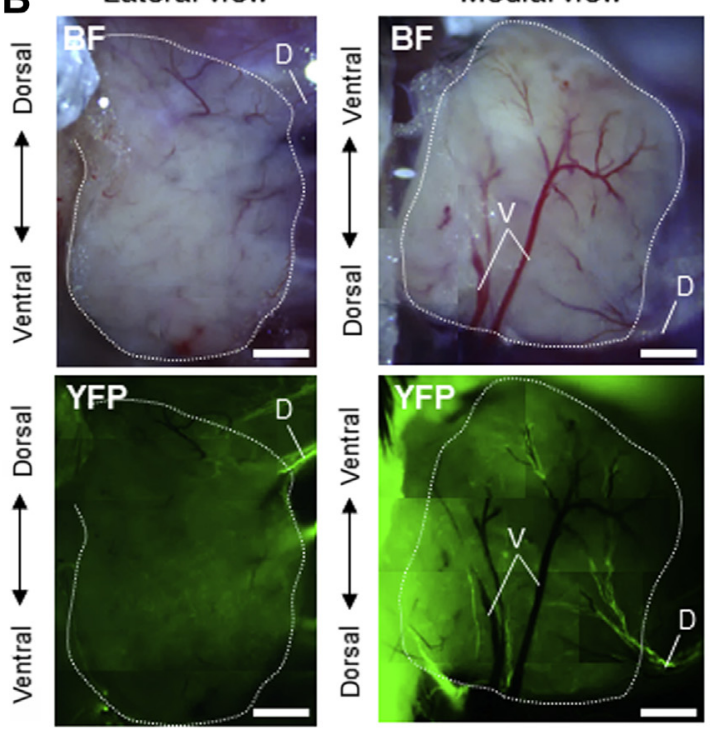

D
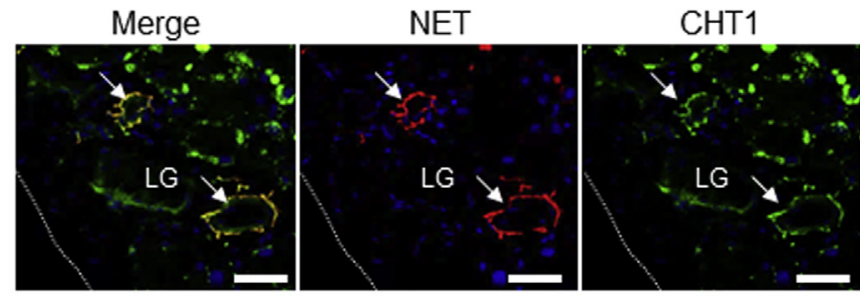

$\mathbf{F}$
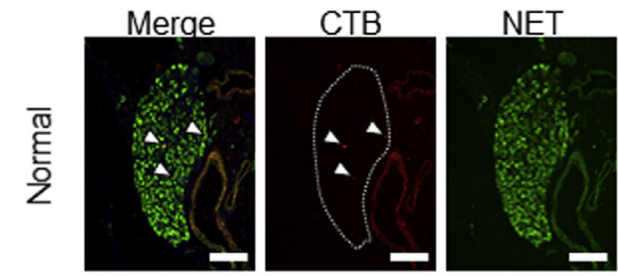

包
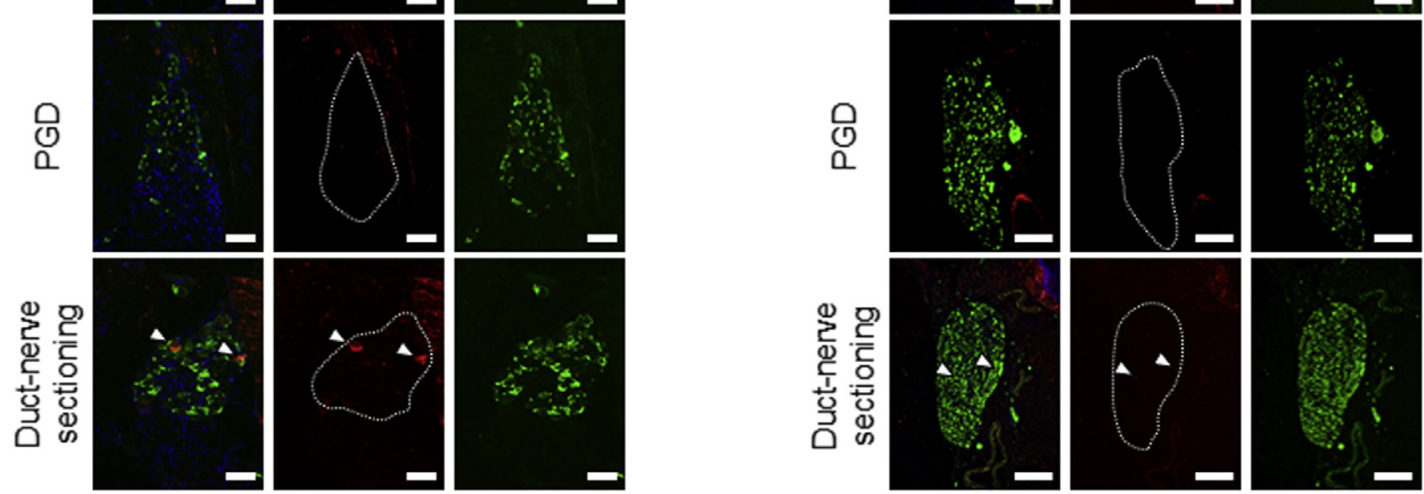

Figure 1 Identification of postganglionic nerves projecting to the lacrimal gland (LG). A: Lateral view and schematic drawing of the right side of a mouse head. B: Bright field (BF) or yellow fluorescent protein (YFP) images of lateral or medial view of the LG in the proteolipid protein-YFP mouse. C: Fluorescent observation (left panel) or schematic drawing (right panel) of the Alexa 555-conjugated cholera toxin subunit B (CTB)-labeled nerve fibers on a medial view of the LG injected with CTB. D: Merged images (left panel), norepinephrine transporter (NET, red; middle panel), and choline transporter-1 (CHT1, green; right panel) immunostaining of a transverse section of the nerve fibers indicated by the orange area in C (right panel). Arrows indicate the NET/CHT1 double-positive nerve fibers. E: Merged images (left column), CTB (red; middle column), and CHT1 immunostaining (green; right column) of the pterygopalatine ganglion (PPG) with CTB injection into the LG. Arrowheads indicate the CTB/CHT1 double-positive neurons. F: Merged images (left column), CTB (red; middle column), and NET immunostaining (green; right column) of the superior cervical ganglion (SCG) with CTB injection into the LG. Arrowheads indicate the CTB/NET double-positive neurons. Dotted lines indicate the LG. Scale bars: $4 \mathrm{~mm}(\mathbf{A}) ; 1 \mathrm{~mm}(\mathbf{B}) ; 400 \mu \mathrm{m}$ (C); $50 \mu \mathrm{m}$ (D and E); $200 \mu \mathrm{m}$ (F). D, duct; N, nerve bundle; V, blood vessel. 
A

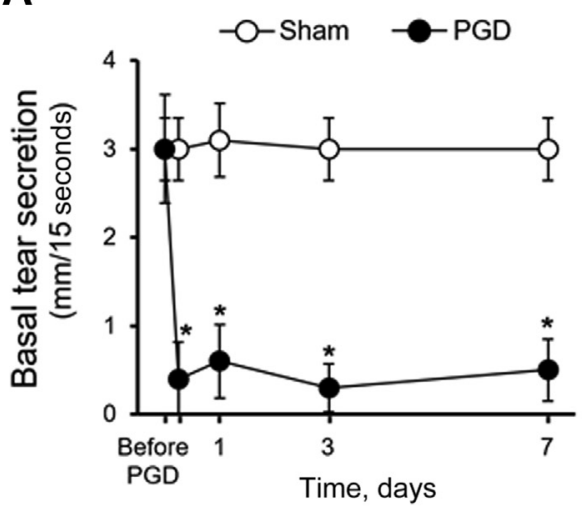

B

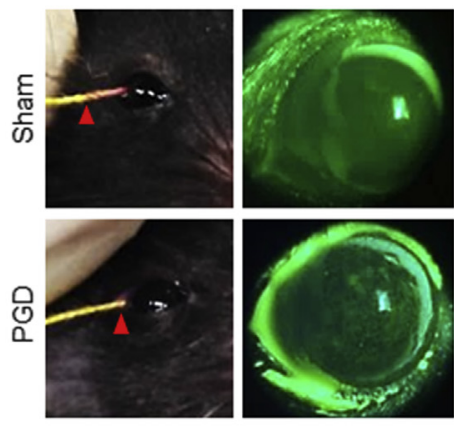

C

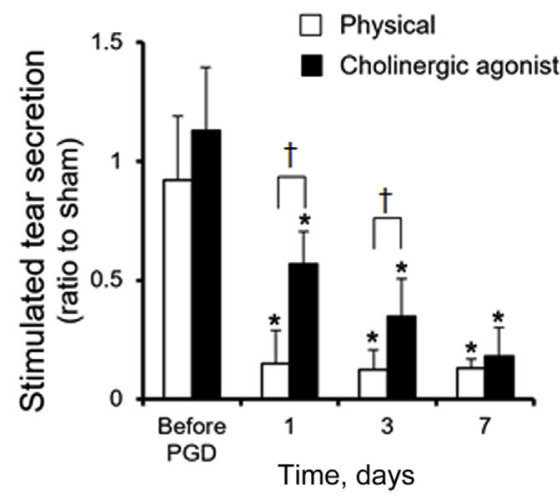

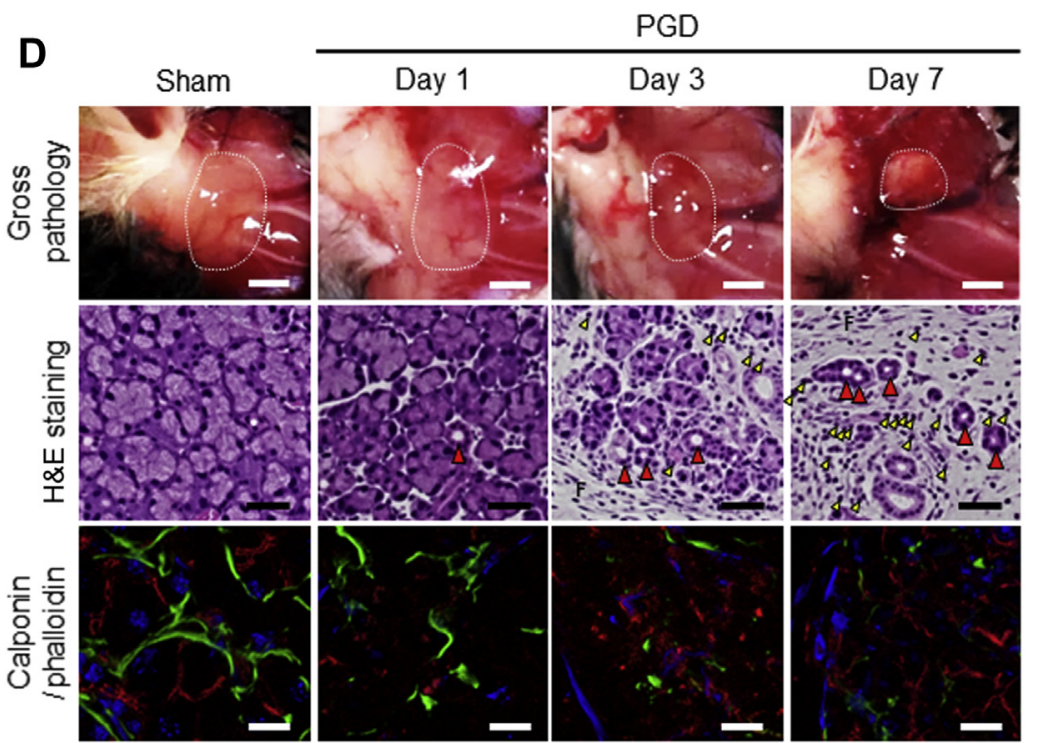

E

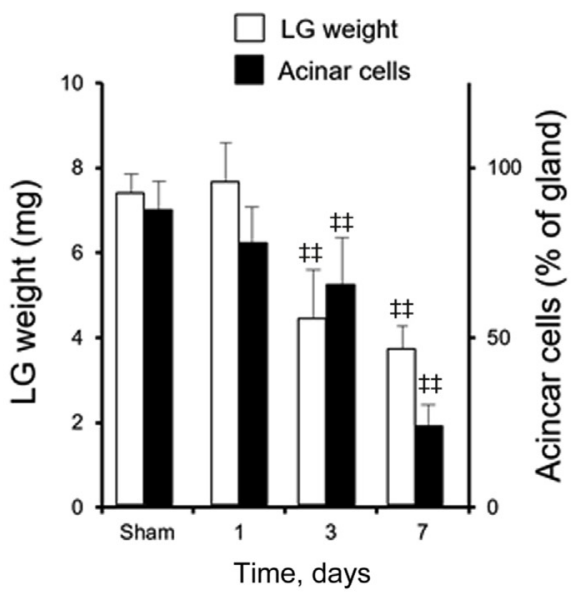

Figure 2 Effect of postganglionic denervation (PGD) on tear secretion. A: Change in basal tear secretion after PGD or sham surgery. B: Representative images of the measurement of basal tear secretion and corneal fluorescein staining. Arrowheads indicate the wetted length due to tear secretion. C: Changes in stimulated tear secretion caused by a physical stimulation or cholinergic agent injection after PGD surgery. D: Gross pathology [dotted lines indicate the lacrimal gland (LG), hematoxylin and eosin (H\&E) staining, and calponin (green) and phalloidin (red) immunostaining of the LG after PGD surgery]. Red arrowheads indicate the enlargement and hyperplasia of ducts; yellow arrowheads, inflammatory cell infiltration. E: Changes in LG weight and acinar cell area after PGD surgery. Data are expressed as means \pm SD. $n=4$ to 9 mice $(\mathbf{A}, \mathbf{C}$, and $\mathbf{E})$. ${ }^{*} P<0.05$ versus before $\mathrm{PGD} ;{ }^{\dagger} P<0.05 ;{ }^{\ddagger \ddagger} P<0.01$ versus sham. Scale bars: $2 \mathrm{~mm}$ (D, top row); $50 \mu \mathrm{m}$ (D, middle row); $25 \mu \mathrm{m}$ (D, bottom row). Original magnification, $\times 2$ (B). F, interstitial fibrogenesis.

were observed caudally in the medial surface of the LG (Figure 1C). These results suggest that the LG receives abundant projection from the nerve bundles distributed along the caudal blood vessels in the medial surface of the LG.

The LG is innervated by the central nervous system through sympathetic and parasympathetic nerves by way of the superior cervical ganglion and pterygopalatine ganglion, respectively. ${ }^{13}$ To confirm that the nerve bundles identified as projecting into the LG consisted of sympathetic and parasympathetic nerves, immunostaining of the transverse section of these nerve bundles was conducted using sympathetic and parasympathetic markers. Both the sympathetic marker norepinephrine transporter and parasympathetic marker choline transporter-1 were expressed in the nerve bundles (Figure 1D). These results indicate that the identified nerve bundles projecting into the LG consist of sympathetic and parasympathetic nerve fibers.

Moreover, to confirm the connection between ganglions and the nerve bundles thus identified, CTB retrograde labeling was performed in PGD animals. In control animals, $\mathrm{CTB} /$ choline transporter-1 or $\mathrm{CTB} /$ norepinephrine transporter double-positive neurons were observed in the pterygopalatine ganglion and the superior cervical ganglion, respectively (Figure 1, E and F). However, in PGD animals, $\mathrm{CTB} /$ choline transporter-1 or $\mathrm{CTB} /$ norepinephrine transporter double-positive neurons in each ganglion were almost absent (Figure 1, E and F). In contrast to PGD animals, $\mathrm{CTB} /$ choline transporter-1 and $\mathrm{CTB} /$ norepinephrine transporter double-positive neurons were observed in the 
A

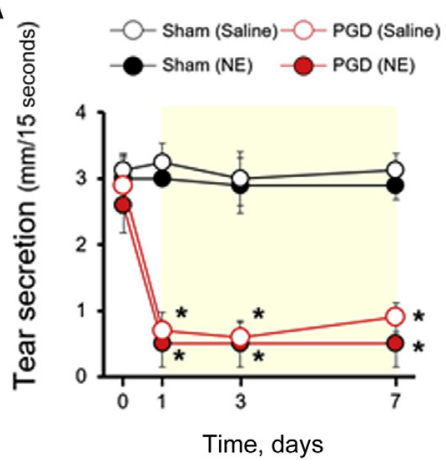

C

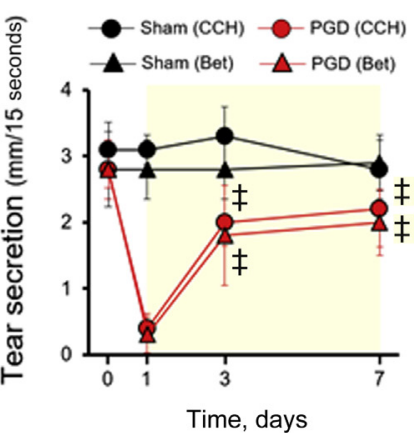

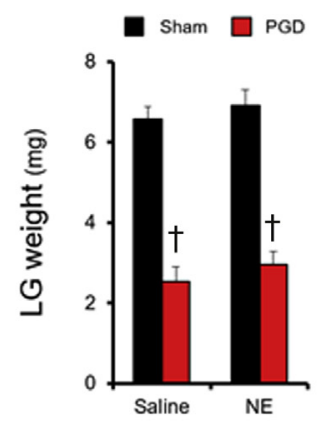

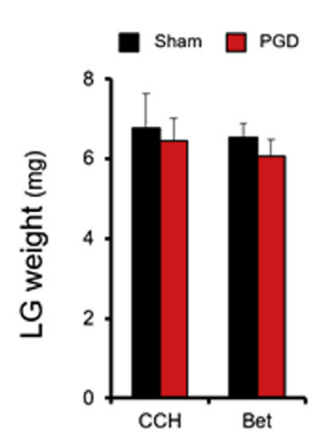

B

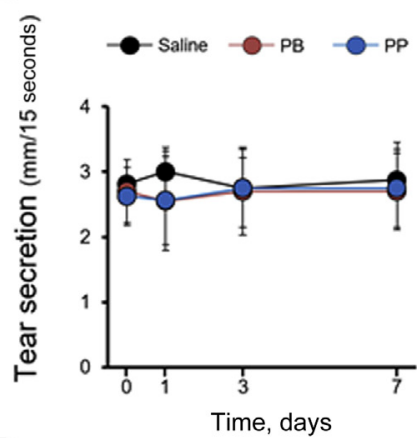

D

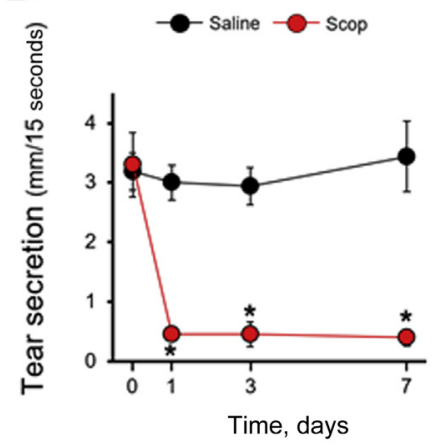

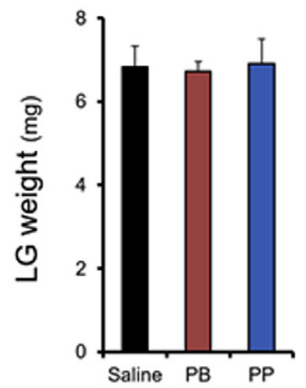

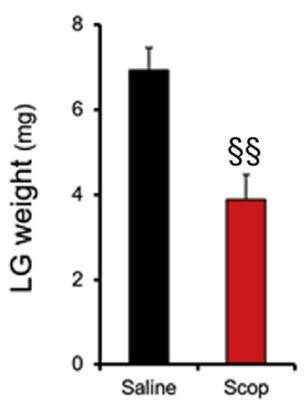

$\mathbf{E}$
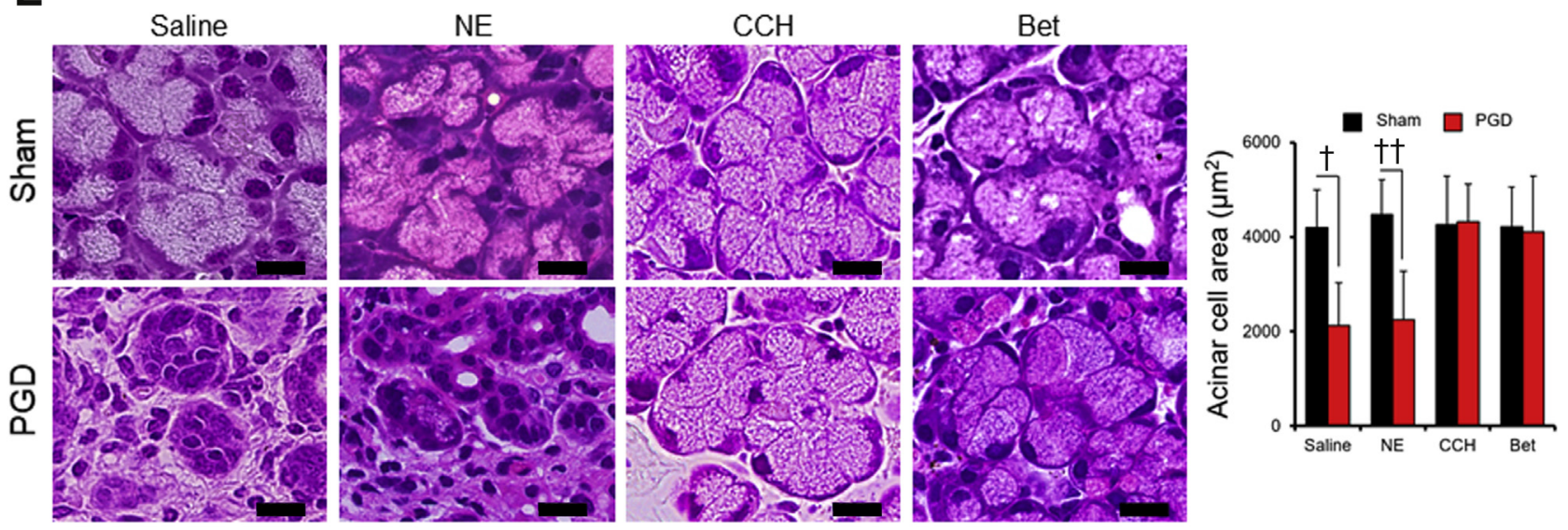

$\mathbf{F}$

Saline
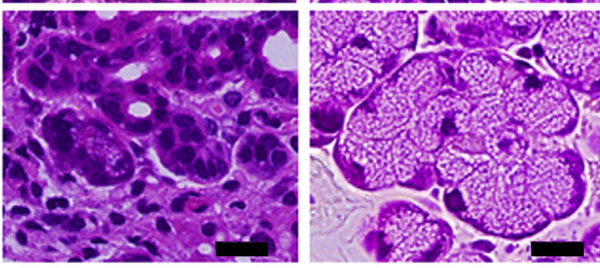

PB
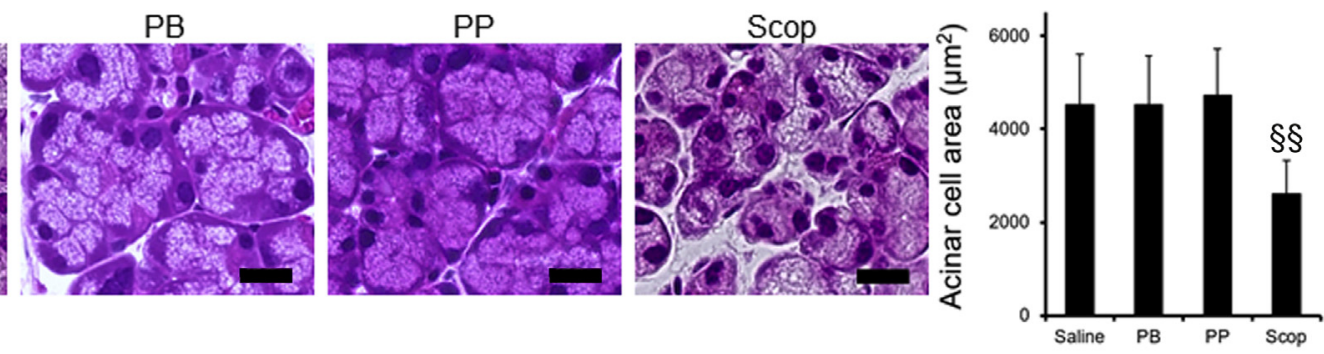

Figure 3 Effect of pharmacologic modulation of autonomic nerve terminals of lacrimal gland (LG). Effect of continuous infusion of a sympathetic agonist (A) or antagonist (B) on tear secretion and LG weight under postganglionic denervation (PGD) or normal state, respectively. Effect of continuous infusion of a parasympathetic agonist (C) or antagonist (D) on tear secretion and LG weight under PGD or normal state, respectively. Hematoxylin and eosin (H\&E) staining of the LG in the mouse with continuous infusion of a sympathetic or parasympathetic agonist under PGD or sham conditions (E) and with continuous infusion of sympathetic or parasympathetic antagonists under a normal state (F). The right bar charts indicate the acinar cell area. Norepinephrine (NE) was used as the sympathetic agonist. Phenoxybenzamine (PB) and propranolol (PP) were used as $\alpha$-adrenoceptor and $\beta$-adrenoceptor antagonists, respectively. Carbachol $(\mathrm{CCH})$ and bethanechol (Bet) were used as nicotinic and muscarinic receptor agonist and as exclusively muscarinic receptor agonist, respectively. Scopolamine (Scop) was used as the muscarinic receptor antagonist. Data are expressed as means \pm SD. $n=4$ to 8 mice $(\mathbf{A}-\mathbf{F})$. ${ }^{*} P<0.05,{ }^{* *} P<0.01$ versus 0 days; ${ }^{\dagger} P<0.05,{ }^{\dagger \dagger} P<0.01$ versus sham; ${ }^{\ddagger} P<0.05$ versus 1 day; ${ }^{\S \S} P<0.01$ versus saline. Scale bars $=10 \mu \mathrm{m}(\mathbf{E}$ and $\mathbf{F})$. 
pterygopalatine ganglion and superior cervical ganglion of duct-sectioned animals, respectively (Figure 1, E and F). These results indicate that postganglionic nerve fibers projected from the pterygopalatine ganglion or the superior cervical ganglion are composed of the nerve bundles identified along the caudal blood vessels and not the rostral lacrimal duct.

\section{Effect of PGD on Tear Secretion}

To investigate the function of the postganglionic nerves thus identified, denervation of postganglionic nerve bundles was performed. Basal tear secretion was significantly decreased immediately after denervation, and this reduction was sustained up to day 7 (Figure 2A), indicating that the
A
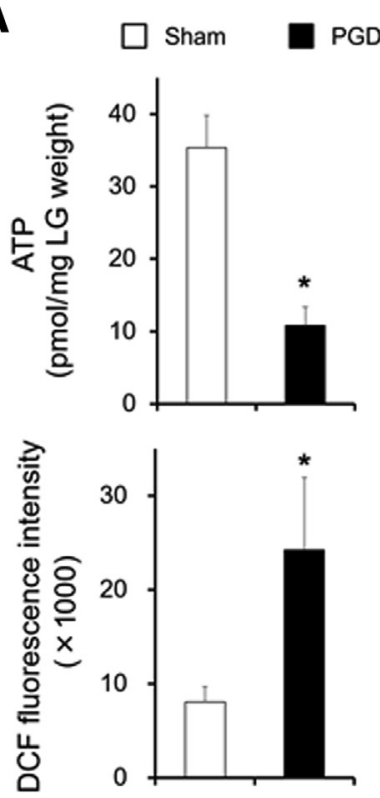

C

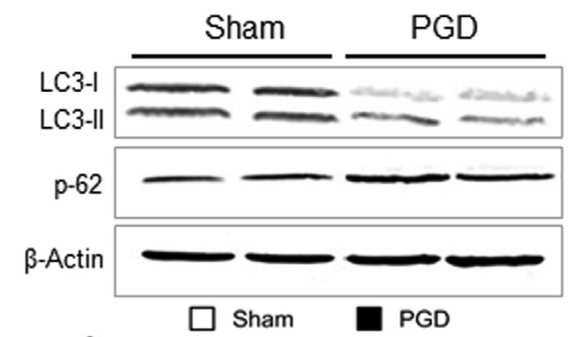

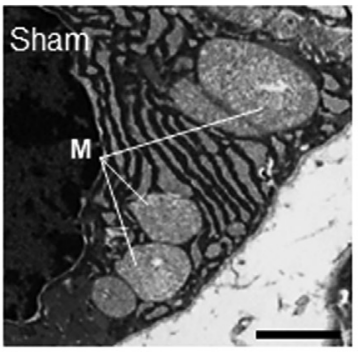

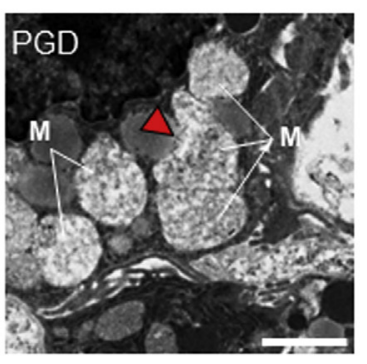

B
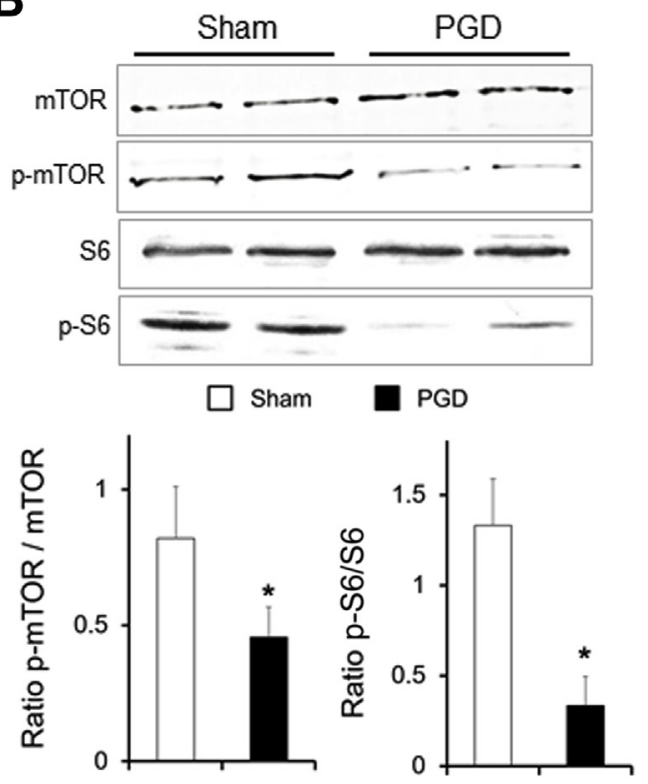
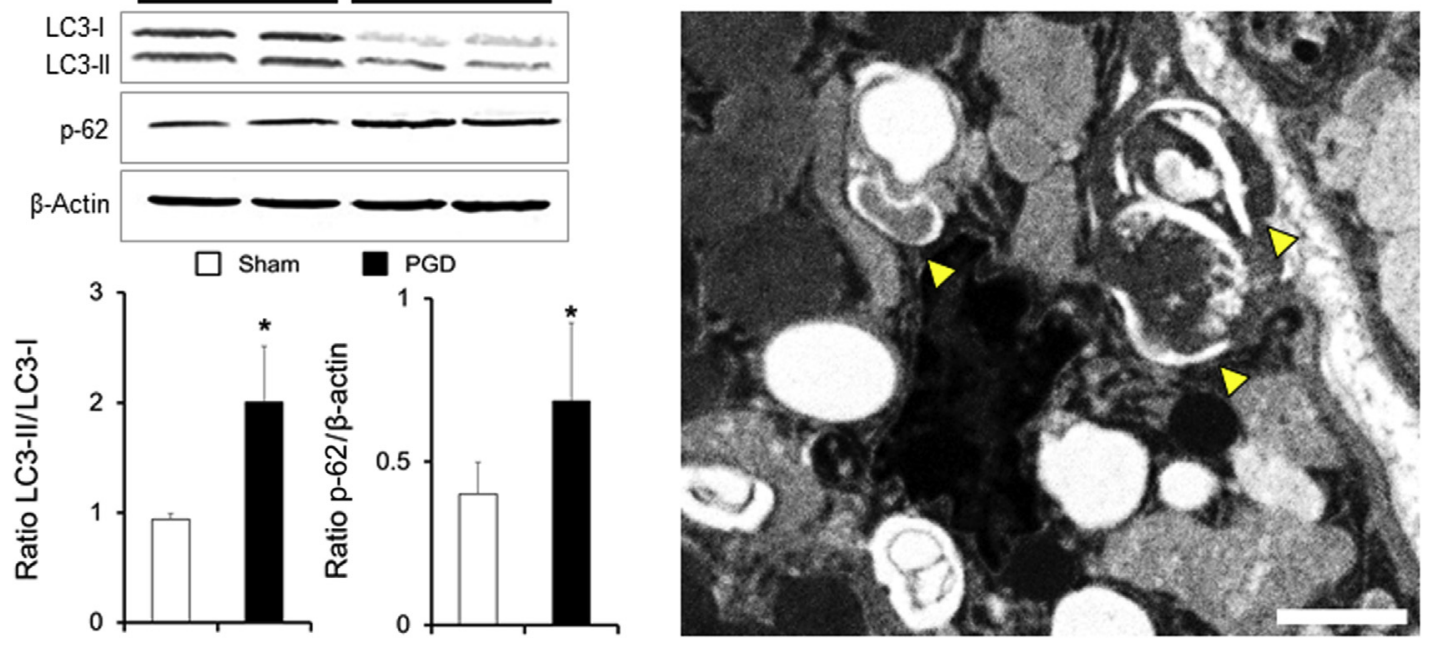

Figure 4 Postganglionic denervation (PGD) lowers the lacrimal gland (LG) energy status and activated autophagy. A: ATP content (top left panel) or dichlorofluorescein fluorescence intensity (bottom left panel) of the LG after PGD surgery or sham. Typical transmission electron microscopy (TEM) observation of mitochondria of an acinar cell in sham (top right panel) and PGD surgery (bottom right panel). Arrowhead indicates mitochondrial swelling. B: Western blotting for mammalian target of rapamycin (mTOR), phospho-mTOR (p-mTOR), ribosomal protein S6 (S6), and phospho-S6 (p-S6) in the LG after PGD surgery (top panel). Ratio of p-mTOR/mTOR (bottom left panel) or p-S6/S6 (bottom right panel) in the LG after PGD surgery or sham. C: Western blotting for p-62, light chain 3 (LC3)-I, LC3-II, and $\beta$-actin in the LG after PGD surgery (top left panel). Ratio of p-62/ $\beta$-actin (bottom left panel) or LC3-II/LC3-I (bottom middle panel) in the LG after PGD or sham surgery. Typical TEM observation of autophagosome formation in an acinar cell after PGD surgery (right panel). Arrowheads indicates autophagosome formation. Data are expressed as means \pm SD. $n=5 \mathrm{LGs}(\mathbf{A}-\mathbf{C})$. ${ }^{*} P<0.05$ versus sham. Scale bars $=1$ $\mu \mathrm{m}$ (A and C). M, mitochondria. 
innervation through ganglions maintains basal tear secretion. Superficial punctate keratopathy was seen everywhere in the cornea after PGD (Figure 2B), indicating that the decrease in basal tear secretion by PGD induced corneal epithelial disorder. Furthermore, physically stimulated secretion after PGD was abolished at all time points, presumably because of the loss of the reflex circuit (Figure 2C). On the other hand, cholinergic agonist-stimulated tear secretion, which is induced by direct stimulation of the M3 acetylcholine receptors on the acinar cells in the LG, decreased but remained observable up to 3 days after PGD, indicating that acetylcholine receptor-mediated tear secretion was retained at these time points (Figure 2C). Seven days after PGD, however, acetylcholine receptor-mediated tear secretion was also abolished. These data indicate that the identified postganglionic nerve bundles play a critical role in basal and physically stimulated tear secretion.

To understand why acetylcholine receptor-mediated tear secretion was eventually lost after PGD, the LG was examined macroscopically (Figure 2, D and E). Atrophic changes in the LG gradually progressed after denervation, and the size of the LG became approximately half that before denervation on day 7 (Figure 2D). Similarly, LG weight was gradually decreased 3 days after PGD, and the weight at 7 days after PGD was significantly decreased (by approximately 50\%) compared with the weight before PGD (Figure 2E). Histologic analysis showed slight atrophy already on day 1 . The acinar cells area ratio was evidently reduced on day 3 and greatly reduced on day 7 (Figure 2E). Only ductal cells remained, and fibroblastic tissue accompanied by inflammatory cell infiltration (largely monocyte) occupied the remaining space (Figure 2E). The number of myoepithelial cells, located on the basal side of acinar cells, was slightly reduced on day 1 and was severely diminished on days 3 and 7 (Figure 2E). These data demonstrate that PGD resulted in the destruction of the tear secretion apparatus.

Because the LG postganglionic nerve bundles are associated with the branch of the superficial temporal artery, it is possible that PGD caused LG ischemia and thus resulted in LG atrophy. To examine this, LG blood flow was measured before and after PGD. Laser Doppler measurement showed that PGD surgery did not alter the LG blood flow (Supplemental Figure S1, B and C). This result indicates that the decrease in tear secretion and the destruction of the LG structure by PGD were not induced by LG ischemia and suggests that the innervation from ganglions to the LG plays a critical role in reflex tear secretion and in maintaining basal tear secretion by supporting the lobular LG structure.

\section{Effect of the Pharmacologic Modulation of the LG Autonomic Nerve Terminals}

The sympathetic $\alpha$ - and $\beta$-adrenoceptor and the parasympathetic M3 acetylcholine receptor are expressed in the acinar cells of the LG. ${ }^{27}$ To dissect the roles of the sympathetic and parasympathetic innervations of the LG, we examined the induced improvement or deterioration in tear secretion and LG atrophy, respectively, by sympathetic or parasympathetic agonists under PGD or by sympathetic or parasympathetic antagonists in the normal state.

The continuous infusion of norepinephrine did not change the tear secretion induced by PGD surgery, and the LG weight in PGD with norepinephrine was significantly lower than that in the sham group (Figure 3A). The continuous infusion of neither the $\alpha$-adrenoceptor antagonist phenoxybenzamine nor the $\beta$-adrenoceptor antagonist propranolol modified tear secretion or LG weight in the normal state (Figure 3B). Similarly, norepinephrine infusion under PGD failed to affect acinar cell size compared with PGD with saline infusion (Figure 3E), and phenoxybenzamine or propranolol infusion did not lead to changes in acinar cell size (Figure 3F). These results indicate that the sympathetic nerves are not involved in the alteration of LG function by PGD.

In addition, to confirm the lack of a role of the sympathetic nerves in tear secretion, superior cervical ganglion ablation was performed, which did not alter tear secretion compared with sham (Supplemental Figure S2B). This result indicates that the sympathetic postganglionic nerves projecting to LG are not related to tear secretion.

In contrast, infusion of the nicotinic and muscarinic acetylcholine receptor agonist carbachol improved tear secretion (Figure 3C) and maintained the LG weight (Figure 3C) and morphology (Figure 3E) under PGD at the same level as in sham surgery. On infusion of the muscarinic acetylcholine receptor agonist bethanechol, improvement and maintenance of tear secretion and LG weight were observed at the same level as that with carbachol infusion under PGD (Figure 3C), indicating that muscarinic and not nicotinic acetylcholine receptors play an important role in the maintenance of LG function and morphology. In agreement with a previous study, ${ }^{28}$ the infusion of the muscarinic acetylcholine receptor antagonist scopolamine reduced tear secretion (Figure 3D) and LG weight (Figure 3D) and induced LG atrophy (Figure 3F) in the normal state at the same levels as those induced by PGD. These results suggest that the alterations of LG function induced by PGD are due to the interruption of parasympathetic neural input to the LG through muscarinic acetylcholine receptors.

\section{PGD Lowered LG Energy Status and Activated Autophagy}

In response to nutrition/energy deprivation, the autophagy pathway is activated to supply cellular energy by accelerating net cellular protein catabolism, which results in organ atrophy. ${ }^{29}$ Parasympathetic acetylcholine generates mitochondria biogenesis and ATP production by increasing cytosolic $\mathrm{Ca}^{2+}$. Changes in the LG energy status and autophagy pathway induced by PGD were evaluated to 
clarify the association between progressive atrophy and interruption of the parasympathetic stimulation of the LG.

To evaluate the LG energy status after PGD, ROS formation and ATP content were examined. A significant decrease in ATP content (Figure 4A) and an increase in ROS formation (Figure 4A) were observed compared with sham. These results indicate that the mitochondrial damage induced by ROS accumulation decreased the ATP content.

To confirm that PGD induces mitochondrial damage in the LG, TEM analysis of the LG was conducted after PGD. Grossly swollen mitochondria were found in the acinar cells of the LG after PGD compared with sham (Figure 4A). These results indicate that PGD actually induces mitochondrial damage in the LG.

The mTOR kinase, a sensor of cellular nutritional status, is an essential mediator of protein synthesis and energy metabolism and is controlled by intracellular ATP. The depletion of ATP inhibits mTOR signaling. ${ }^{30}$ mTOR and S6 proteins were analyzed to evaluate the cellular nutritional status in the LG after PGD. PGD of LG significantly decreased both $\mathrm{p}$-mTOR/mTOR and p-S6/S6 ratios compared with sham (Figure 4B). These results indicate that PGD deteriorates the cellular nutritional status in the LG through the inhibition of mTOR signaling.

To evaluate the level of autophagy, the autophagy protein LC3 and, again, mTOR, which is a widely used marker of mammalian autophagy, were analyzed. During autophagy, the LC3-I protein localized in the cytoplasm is cleaved, lipidated, and inserted as LC3-II into the autophagosome membrane. ${ }^{31}$ PGD of LG significantly increased the LC3-II/ LC3-I and the p62/ $\beta$-actin ratios (Figure $4 \mathrm{C}$ ). These results suggest an increase of autophagy levels in the LG after PGD. On TEM analysis, autophagosome formations were found in the acinar cells of the LG after PGD (Figure 4C). These results indicated that activation of the autophagy process is involved in LG atrophy induced by PGD.

\section{Discussion}

Tear secretion is primarily under the control of the sensory, sympathetic, and parasympathetic nervous systems. ${ }^{2}$ In agreement with previous work, this study demonstrated that parasympathetic stimulation plays a role in maintaining LG secretory function. ${ }^{17,32-34}$ In contrast, the impairment of sympathetic neural input by surgical denervation or pharmacologic modulation did not alter tear secretion or LG morphology, suggesting that sympathetic innervation has little effect on the mouse LG function. It is widely acknowledged that the parasympathetic innervation of the LG is responsible for tear secretion, although its role has not been fully elucidated yet. Tangkrisanavinont ${ }^{15}$ reported that the electrical stimulation of the superior cervical ganglion induced tear secretion from the rabbit LG. Ruskell ${ }^{17}$ and Meneray et $\mathrm{al}^{32}$ reported that the surgical removal of the superior cervical ganglion did not alter tear secretion and
LG morphology in the monkey and rabbit, respectively. The superior cervical ganglion provides sympathetic innervation to not only the LG but also the pineal gland, the blood vessels in the cranial muscles and brain, the choroid plexus, the eyes, the carotid body, the salivary glands, and the thyroid gland. ${ }^{35,36}$ These discrepancies may therefore be explained by the complex influence of sympathetically innervated areas other than the LG. Our approach, based on the denervation of the postganglionic nerves specifically innervating the LG, differs from previous related work attempting to demonstrate the exact role of sympathetic innervation of the LG. ${ }^{33,34}$

Organ atrophy is characterized by a decrease in the tissue mass of an organ due to a decrease in the size of the individual cells and/or in their number. ${ }^{37}$ Disuse (ie, reduced activity mediated by a reduction of neural signals) is the major cause of atrophy. ${ }^{38,39}$ In the salivary gland, which has a secretory mechanism analogous to the LG, disuse atrophy is induced by reduced reflex stimulation for salivary secretion, normally generated in response to masticatory activity. ${ }^{40-42}$ These observations with PGD, namely the loss of the ability to secrete tears together with acute LG atrophy, can be explained as a consequence of disuse degeneration induced by blocking the LG parasympathetic stimulation.

Under this atrophic condition, net protein and lipid catabolism is accelerated in response to an impaired cellular energy status. ${ }^{43}$ Cellular energy homeostasis is regulated by a complex physiologic network. ${ }^{44}$ Autophagy is a process of lysosomal protein degradation of long-lived proteins and cytoplasmic organelles mediated by autophagosomes, vesicles characterized by a double-layer membrane. ${ }^{31,44,45}$ In addition, autophagy acts as a supply of cellular energy in response to nutritional restriction. ${ }^{46}$ Amino acids resulting from this process can be used directly to drive ATP conversion to Krebs cycle intermediates. ${ }^{47}$ These processes can be activated through the inactivation of mTOR, which is a key integrator of signals from nutrients and energy status. ${ }^{48}$ Parasympathetic cholinergic stimuli elicit an IP3-dependent $\left[\mathrm{Ca}^{2+}\right] \mathrm{i}$ increase in $\mathrm{LG}$ acinar cells via the activation of M3 muscarinic acetylcholine receptor, ${ }^{25}$ and an increase in $\left[\mathrm{Ca}^{2+}\right] \mathrm{i}$ is known to activate the mTOR pathway. ${ }^{49}$ Thus, this investigation e speculates that the LG atrophy induced by the interruption of parasympathetic stimuli presented in the current study is triggered by a decrease in $\left[\mathrm{Ca}^{2+}\right] \mathrm{i}$, leading to impaired cellular energetics and activation of an autophagic pathway through the inhibition of mTOR phosphorylation.

The characteristic features of the changes in LG function after the interruption of parasympathetic stimuli by PGD were an immediate decrease in tear secretion accompanied by LG atrophy, with destruction of the lobular structure. Parasympathetic nerves originating from the superior salivatory nucleus project to the LG through the preganglionic greater superficial petrosal nerve (GSPN) and postganglionic nerves, with pterygopalatine ganglion as an intermediate. ${ }^{13}$ Nguyen et $\mathrm{al}^{33}$ performed GSPN sectioning, 
with a surgical approach from the internal auditory meatus to the GSPN, located in front of the petrous temporal bone, to investigate the effect of a series of parasympathetic stimuli on the LG. ${ }^{34}$ They reported that changes in the LG were limited to the up-regulation of proinflammatory genes and the accumulation of secretory granules in acinar cells. Once a nerve axon is injured, it is disconnected from the cell body and eventually degenerates, although this degeneration does not proceed transneuronally. ${ }^{50}$ Therefore, we could speculate that, in contrast to the PGD performed here, the neural degeneration induced by GSPN sectioning was limited to this preganglionic nerve, and the postganglionic nerve projecting to LG remained unaltered. The discrepancy between the effects of GSPN sectioning and the PGD of the current study on the LG was presumably due to the effect of postganglionic nerve activities, which were respectively maintained or compromised by GSPN sectioning or PGD. Furthermore, the results of the pharmacologic blockade of the parasympathetic nerve terminals of the LG showed that the morphologic alteration of the LG was identical to that induced by PGD. These results suggest that PGD can identify more precisely the effect of parasympathetic stimuli on LG function by excluding neural activity other than that of postganglionic nerves, with minimal surgical insult or systemic pharmacologic effects.

It was previously reported, in a human epidemiologic study and rat blink-suppressed dry eye model, that LG dysfunction contributes to technological device-associated dry eye. 5 However, the mechanisms involved in the occurrence of LG dysfunction remains unclear. Blinking plays an important role in maintaining basal tear secretion. On continuous blinking, sensory signals evoked by the friction between the corneal surface and the eyelid are persistently transmitted to the LG through sensory and parasympathetic nerves. $^{8}$ In the present study on LG dysfunction, chronic reduction in tear secretion and morphologic destruction of the LG were induced by a loss of parasympathetic stimuli, suggesting that the suppressed parasympathetic stimulation of the LG associated with reduced blinking is a critical mechanism that leads to this type of dry eye.

This study has shown, to our knowledge for the first time, that postganglionic nerves project to the LG. In addition, the role of parasympathetic innervation in tear secretion by the denervation of this nerve is confirmed. Furthermore, parasympathetic simulation is essential in maintaining the energy status of the LG secretory function. Further investigations focused on autonomic nerve regulation of the LG secretory function may contribute to the prevention and management of dry eye.

\section{Acknowledgment}

We thank the Collaborative Research Resources, School of Medicine, Keio University for technical assistance.

\section{Author Contributions}

S.N., M.I., and K.F.T. designed the research; K.J., T.I., and R.H. performed animal experiments; M.I. performed TEM analysis; K.J. and T.I. analyzed the data and wrote the original manuscript; S.N. and K.T. edited the manuscript; H.T. performed blood flow analysis; K.F.T. generated transgenic mice.

\section{Supplemental Data}

Supplemental material for this article can be found at http://doi.org/10.1016/j.ajpath.2020.01.007.

\section{References}

1. Murube J: Basal, reflex, and psycho-emotional tears. Ocul Surf 2009, 7:60-66

2. Dartt DA: Neural regulation of lacrimal gland secretory processes: relevance in dry eye diseases. Prog Retin Eye Res 2009, 28:155-177

3. Frumin I, Rozenkrantz L, Gelstein S, Sobel N, Roth Y, Yeshurun Y, Shushan S: Human tears contain a chemosignal. Science 2011, 331: 226-230

4. Kawashima M, Uchino M, Yokoi N, Uchino Y, Dogru M, Komuro A, Sonomura Y, Kato H, Nishiwaki Y, Kinoshita S, Tsubota K: The association between dry eye disease and physical activity as well as sedentary behavior: results from the Osaka study. J Ophthalmol 2014, 2014:943786

5. Nakamura S, Shibuya M, Nakashima H, Imagawa T, Uehara M, Tsubota K: D- $\beta$-hydroxybutyrate protects against corneal epithelial disorders in a rat dry eye model with jogging board. Invest Ophthalmol Vis Sci 2005, 46:2379-2387

6. Nakamura S, Kinoshita S, Yokoi N, Ogawa Y, Shibuya M, Nakashima H, Hisamura R, Imada T, Imagawa T, Uehara M, Shibuya I, Dogru M, Ward S, Tsubota K: Lacrimal hypofunction as a new mechanism of dry eye in visual display terminal users. PLoS One 2010, 5:e11119

7. Rushworth G: Observations on blink reflexes. J Neurol Neurosurg Psychiatry 1962, 25:93-108

8. Rolando M, Zierhut M: The ocular surface and tear film and their dysfunction in dry eye disease. Surv Ophthalmol 2001, 45 Suppl 2: S203-S210

9. Jordan A, Baum J: Basic tear flow: does it exist? Ophthalmology 1980, 87:920-930

10. Kamoi M, Ogawa Y, Nakamura S, Dogru M, Nagai T, Obata H, Ito M, Kaido M, Kawakita T, Okada Y, Kawakami Y, Shimmura S, Tsubota K: Accumulation of secretory vesicles in the lacrimal gland epithelia is related to non-Sjögren's type dry eye in visual display terminal users. PLoS One 2012, 7:e43688

11. Dartt DA: Dysfunctional neural regulation of lacrimal gland secretion and its role in the pathogenesis of dry eye syndromes. Ocul Surf 2004, 2:76-91

12. Stern ME, Gao J, Siemasko KF, Beuerman RW, Pflugfelder SC: The role of the lacrimal functional unit in the pathophysiology of dry eye. Exp Eye Res 2004, 78:409-416

13. Tóth IE, Boldogkői Z, Medveczky I, Palkovits M: Lacrimal preganglionic neurons form a subdivision of the superior salivatory nucleus of rat: transneuronal labelling by pseudorabies virus. J Auton Nerv Syst 1999, 77:45-54

14. van der Werf F, Baljet B, Prins M, Otto JA: Innervation of the lacrimal gland in the cynomolgous monkey: a retrograde tracing study. J Anat 1996, 188:591-601 
15. Tangkrisanavinont V: Stimulation of lacrimal secretion by sympathetic nerve impulses in the rabbit. Life Sci 1984, 34:2365-2371

16. Botelho SY, Hisada M, Fuenmayor N: Functional innervation of the lacrimal gland in the cat. Origin of secretomotor fibers in the lacrimal nerve. Arch Ophthalmol 1966, 76:581-588

17. Ruskell GL: Changes in nerve terminals and acini of the lacrimal gland and changes in secretion induced by autonomic denervation. Z Zellforsch Mikrosk Anat 1969, 94:261-281

18. Uddman R, Malm L, Sundler F: The origin of vasoactive intestinal polypeptide (VIP) nerves in the feline nasal mucosa. Acta Otolaryngol 1980, 89:152-156

19. LeDoux MS, Zhou Q, Murphy RB, Greene ML, Ryan P: Parasympathetic innervation of the meibomian glands in rats. Invest Ophthalmol Vis Sci 2001, 42:2434-2441

20. Cuthbertson S, LeDoux MS, Jones S, Jones J, Zhou Q, Gong S, Ryan P, Reiner A: Localization of preganglionic neurons that innervate choroidal neurons of pterygopalatine ganglion. Invest Ophthalmol Vis Sci 2003, 44:3713

21. Salvatore MF, Pedroza L, Beuerman RW: Denervation of rabbit lacrimal gland increases levels of transferrin and unidentified tear proteins of 44 and $36 \mathrm{kDa}$. Curr Eye Res 1999, 18:455-466

22. Inamura $\mathrm{N}$, Sugio $\mathrm{S}$, Macklin WB, Tomita $\mathrm{K}$, Tanaka KF, Ikenaka K: Gene induction in mature oligodendrocytes with a PLP-tTA mouse line. Genesis 2012, 50:424-428

23. Sakamoto R, Bennett ES, Henry VA, Paragina S, Narumi T, Izumi Y, Kamei Y, Nagatomi E, Miyanaga Y, Hamano H: The phenol red thread tear test: a cross-cultural study. Invest Ophthalmol Vis Sci 1993, 34:3510-3514

24. Koh S, Watanabe H, Hosohata J, Hori Y, Hibino S, Nishida K, Maeda N, Tano Y: Diagnosing dry eye using a blue-free barrier filter. Am J Ophthalmol 2003, 136:513-519

25. Dartt DA: Signal transduction and control of lacrimal gland protein secretion: a review. Curr Eye Res 1989, 8:619-636

26. Kawamoto T, Shimizu M: A method for preparing 2- to 50- $\mu$ m-thick fresh-frozen sections of large samples and undecalcified hard tissues. Histochem Cell Biol 2000, 113:331-339

27. Ding C, Walcott B, Keyser KT: Sympathetic neural control of the mouse lacrimal gland. Invest Ophthalmol Vis Sci 2003, 44:1513

28. Dursun D, Wang M, Monroy D, Li DQ, Lokeshwar BL, Stern ME, Pflugfelder SC: A mouse model of keratoconjunctivitis sicca. Invest Ophthalmol Vis Sci 2002, 43:632-638

29. Aucello M, Dobrowolny G, Musarò A: Localized accumulation of oxidative stress causes muscle atrophy through activation of an autophagic pathway. Autophagy 2009, 5:527-529

30. McLeod LE, Proud CG: ATP depletion increases phosphorylation of elongation factor eEF2 in adult cardiomyocytes independently of inhibition of mTOR signalling. FEBS Lett 2002, 531:448-452

31. Kabeya Y: LC3, a mammalian homologue of yeast Apg8p, is localized in autophagosome membranes after processing. EMBO J 2000, 19:5720-5728

32. Meneray MA, Bennett DJ, Nguyen DH, Beuerman RW: Effect of sensory denervation on the structure and physiologic responsiveness of rabbit lacrimal gland. Cornea 1998, 17:99-107

33. Nguyen DH, Toshida H, Schurr J, Beuerman RW: Microarray analysis of the rat lacrimal gland following the loss of parasympathetic control of secretion. Physiol Genomics 2004, 18:108
34. Toshida H, Nguyen DH, Beuerman RW, Murakami A: Evaluation of novel dry eye model: preganglionic parasympathetic denervation in rabbit. Invest Ophthalmol Vis Sci 2007, 48:4468

35. Bowers CW, Dahm LM, Zigmond RE: The number and distribution of sympathetic neurons that innervate the rat pineal gland. Neuroscience 1984, 13:87-96

36. Arbab MAR, Wiklund L, Svendgaard NA: Origin and distribution of cerebral vascular innervation from superior cervical, trigeminal and spinal ganglia investigated with retrograde and anterograde WGA-HRP tracing in the rat. Neuroscience 1986, 19:695-708

37. Riley DA, Ellis S, Slocum GR, Sedlak FR, Bain JL, Krippendorf BB, Lehman CT, Macias MY, Thompson JL, Vijayan K, De Bruin JA: Inflight and postflight changes in skeletal muscles of SLS-1 and SLS-2 spaceflown rats. J Appl Physiol (1985) 2017, 81:133-144

38. O'Leary MFN, Hood DA: Denervation-induced oxidative stress and autophagy signaling in muscle. Autophagy 2009, 5:230-231

39. Muller FL, Song W, Jang YC, Liu Y, Sabia M, Richardson A, Van Remmen H: Denervation-induced skeletal muscle atrophy is associated with increased mitochondrial ROS production. Am J Physiol Regul Integr Comp Physiol 2007, 293: R1159-R1168

40. Hall HD, Schneyer CA: Salivary gland atrophy in rat induced by liquid diet. Exp Biol Med 1964, 117:789-793

41. Scott J, Gunn DL: A comparative quantitative histological investigation of atrophic changes in the major salivary glands of liquid-fed rats. Arch Oral Biol 1991, 36:855-857

42. Scott J, Gunn DL: Functional characteristics of atrophic parotid acinar cells from rats after liquid feeding. J Dent Res 1994, 73:1180-1186

43. Cannavino J, Brocca L, Sandri M, Grassi B, Bottinelli R, Pellegrino MA, Pellegrino MA: The role of alterations in mitochondrial dynamics and PGC-1 $\alpha$ over-expression in fast muscle atrophy following hindlimb unloading. J Physiol 2015, 593: 1981-1995

44. Kuma A, Hatano M, Matsui M, Yamamoto A, Nakaya H, Yoshimori T, Ohsumi Y, Tokuhisa T, Mizushima N: The role of autophagy during the early neonatal starvation period. Nature 2004, 432:1032-1036

45. Mizushima N, Yoshimori T, Ohsumi Y: The role of Atg proteins in autophagosome formation. Annu Rev Cell Dev Biol 2011, 27: $107-132$

46. Tsukamoto S, Kuma A, Murakami M, Kishi C, Yamamoto A, Mizushima N: Autophagy is essential for preimplantation development of mouse embryos. Science 2008, 321:117-120

47. Efeyan A, Zoncu R, Sabatini DM: Amino acids and mTORC1: from lysosomes to disease. Trends Mol Med 2012, 18:524-533

48. Chen J, Fang Y: A novel pathway regulating the mammalian target of rapamycin (mTOR) signaling. Biochem Pharmacol 2002, 64: 1071-1077

49. Gulati P, Gaspers LD, Dann SG, Joaquin M, Nobukuni T, Natt F, Kozma SC, Thomas AP, Thomas G: Amino acids activate mTOR complex 1 via $\mathrm{Ca} 2+/ \mathrm{CaM}$ signaling to hVps34. Cell Metab 2008, 7: 456-465

50. Raff MC, Whitmore AV, Finn JT: Axonal self-destruction and neurodegeneration. Science 2002, 296:868-871 